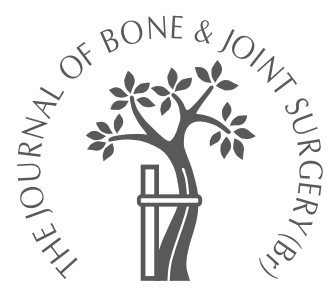

- CASE REPORT

\title{
Treatment of pathological fracture of the femur due to diffuse haemangioma in the lower limb
}

\author{
A REPORT OF TWO CASES
}

\author{
K. Eimori, \\ A. Ogose, \\ T. Hotta, \\ H. Kawashima, \\ G. Omori, \\ N. Endo
}

From Niigata

University, Niigata, Japan

\begin{abstract}
We describe two patients with a diffuse haemangioma of the lower limb complicated by pathological fracture of the femoral shaft, one of whom was treated by a bone graft and immobilisation in a cast, and the other by external fixation and injection of bone marrow. A review of the literature identified difficulty in control of bleeding and obtaining bony union.
\end{abstract}

Haemangioma is a common benign tumour which typically arises in soft tissue. Small lesions may be resected for cosmetic purposes but larger tumours can bleed extensively during surgery. They may involve bone; pathological fractures, although rare, unite poorly and their treatment is often associated with complications. ${ }^{1-4}$ We describe two cases of diffuse haemangioma of the lower limb which were complicated by pathological fracture of the femoral shaft. One patient was treated by a bone graft and hip spica, and the other by external fixation and injection of bone marrow. Delayed union and difficulty in controlling bleeding occurred in both.

\section{Case reports}

Case 1. A 21-year-old woman underwent partial resection of a haemangioma of her left thigh which had been diagnosed at the age of seven years. She sustained a fracture of the left femur as a result of a fall when aged 24. This was treated by closed reduction and application of a hip spica. She sustained a further fracture after falling when aged 43. An external fixator was applied at another hospital and, one week later, she started to walk with the aid of a crutch. Six months later, there was delayed union (Fig. 1a) and she was transferred to our hospital. MRI showed a haemangioma in the anterior portion of the femur. We planned to carry out plate fixation and bone grafting through a posterolateral approach, but on removing the external fixator, massive bleeding occurred from the surface of the bone. Consequently, we inserted an autologous bone graft with hydroxyapatite blocks to the site of the fracture and applied a hip spica cast. The amount of blood lost during surgery was 1600 $\mathrm{ml}$ and the level of haemoglobin fell from 12.1 $\mathrm{g} / \mathrm{dl}$ to $8.6 \mathrm{~g} / \mathrm{dl}$. The patient was transfused with $1200 \mathrm{ml}$ of her own predonated blood. Five months after the procedure (Fig. 1b), the fracture had united, the hip spica cast was removed and a long-leg brace was applied. Ten years later she remains asymptomatic.

Case 2. A 78-year-old woman presented to another hospital with pain in her left thigh after a fall. She described occasional pain in the left lower limb from an early age. Radiographs showed a transverse fracture of the left femoral shaft (Fig. 2a). Open reduction and internal fixation with plating was attempted but on incising the skin and fascia there was blood loss of $3000 \mathrm{ml}$. A diffuse haemangioma was noted and the procedure was abandoned. Radiographs showed sclerotic changes in the femur with some soft-tissue calcification. MRI revealed an extensive haemangioma involving skin, subcutaneous tissue, muscle and bone. She was transferred to our hospital.

Because of the risk of massive blood loss, plating would be difficult and intramedullary nailing impossible because the fracture had partially consolidated and the medullary cavity was narrow. Therefore, we used external fixation. During the procedure the intra-operative blood loss was $20 \mathrm{ml}$, but there was extensive swelling of the thigh and the level of haemoglobin fell from $8.1 \mathrm{~g} / \mathrm{dl}$ to $6.4 \mathrm{~g} / \mathrm{dl}$, the platelet count from $15.5 \times 10^{4} / \mathrm{dl}$ to $9.5 \times 10^{4} / \mathrm{dl}$ and serum levels of fibrin degradation products and D-dimer increased. Four units of blood were transfused.

After external fixation, the patient could walk with two crutches, but radiographs six months later showed delayed union. Since conventional autologous bone grafting would 


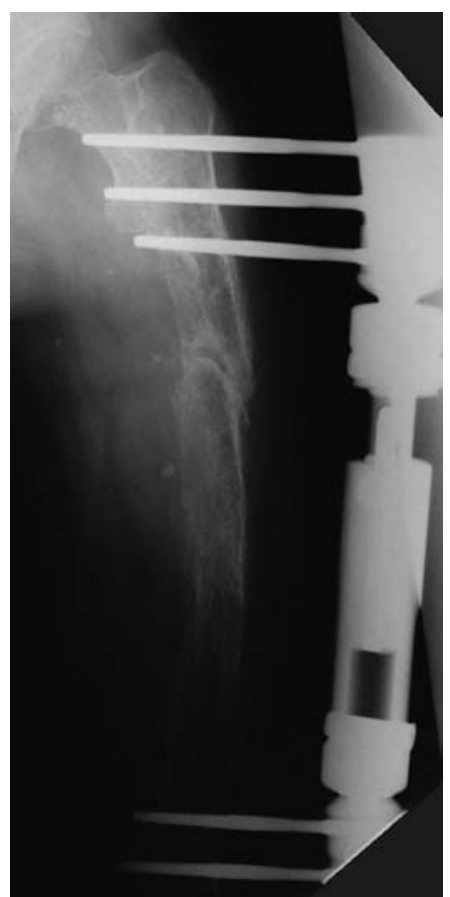

Fig. 1a

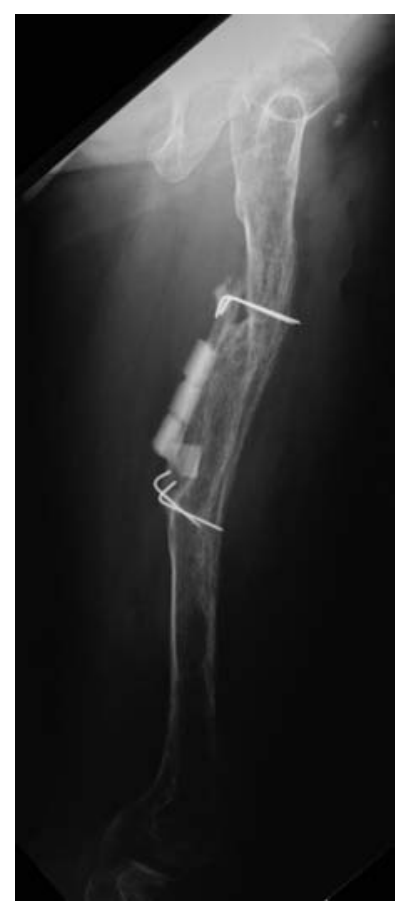

Fig. 1b
Radiographs showing a) nonunion of a pathological fracture of the femur six months after external fixation and b) union five months after bone and hydroxyapatite grafting and application of a hip spica cast.

have caused massive bleeding, we injected bone marrow taken from the iliac crest into the site of the fracture under local anaesthesia. There was minimal bleeding and no change in the level of haemoglobin after the injection. The injection was repeated every two or three months and union progressed gradually (Figs $2 \mathrm{~b}$ and $2 \mathrm{c}$ ).

Six injections were administered. The external fixator was removed two years after the fracture and a walking hip spica cast applied for four weeks. Blood loss at removal of the fixator was $30 \mathrm{ml}$ but the level of haemoglobin fell from $12.4 \mathrm{~g} / \mathrm{dl}$ to $9.9 \mathrm{~g} / \mathrm{dl}$, suggesting bleeding into the thigh. No blood was transfused. After removal of the hip spica, a long-leg brace was applied and the patient was able to walk with a stick. Fourteen months after removal of the external fixator, there was satisfactory bony union and the range of knee movement was from $0^{\circ}$ to $50^{\circ}$. She had no discomfort during daily activities.

\section{Discussion}

There have been few reports on the management of pathological fractures of long bones caused by haemangioma and no consensus on the treatment of choice. We could identify only four cases in the literature..$^{1-4}$

Ezawa et $\mathrm{al}^{3}$ described a five-year-old child who presented with multiple haemangiomas of the femur and tibia which were characterised by repeated pathological fracture. Open reduction and curettage of the tumour under tourniquet control were performed. The fracture united and no bleeding was noted. These haemangiomas arose exclusively in bone without soft-tissue involvement.

Diffuse haemangiomas (angiomatosis) with bone and soft-tissue involvement, similar to our cases, can cause massive blood loss during surgery. Chen et $\mathrm{al}^{4}$ reported a 20 -

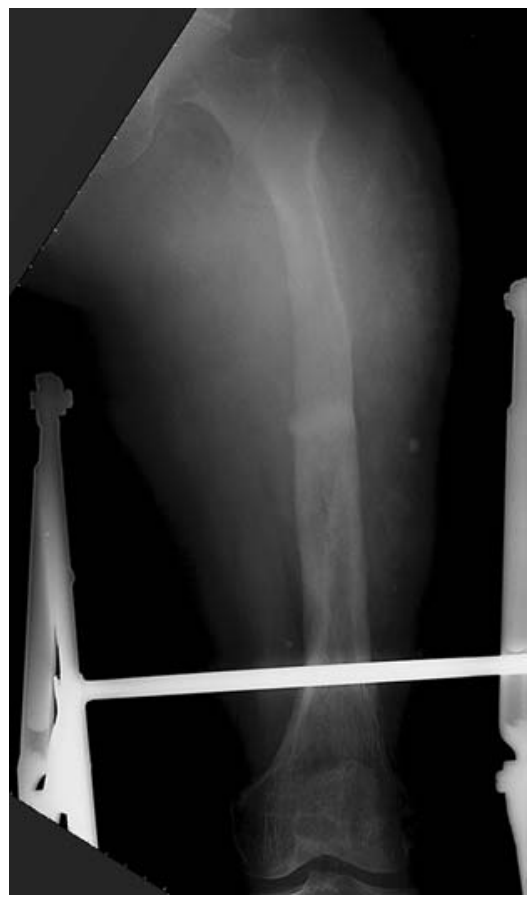

Fig. 2a

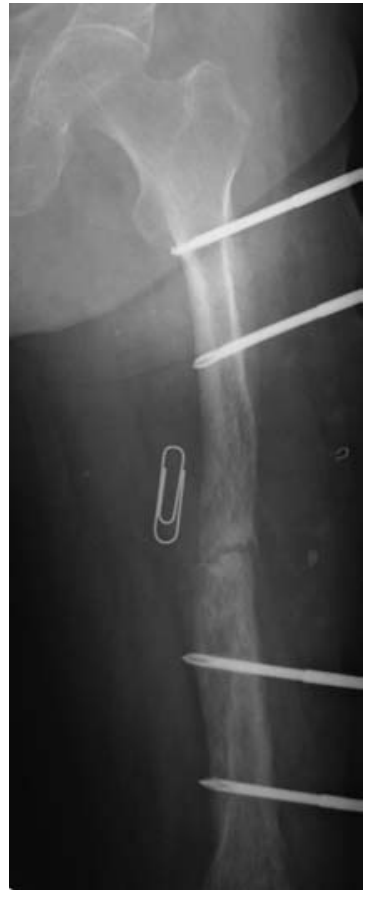

Fig. $2 b$

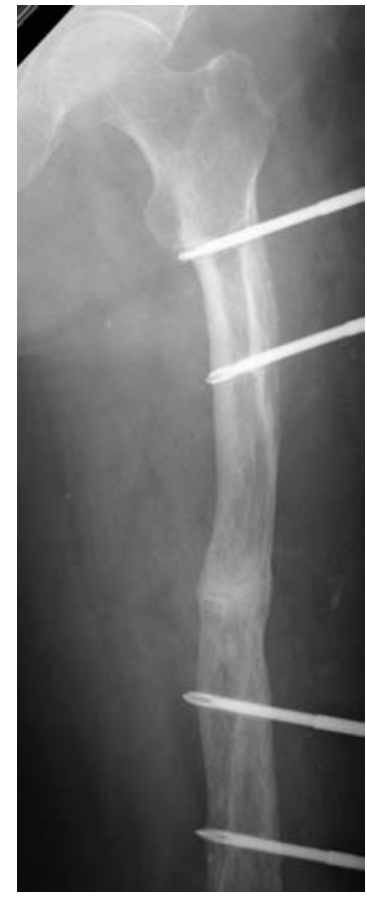

Fig. 2c

Radiographs showing a) a fracture of the femur with soft-tissue calcification, b) the appearance just before injection of bone marrow and c) after six injections. 
year-old woman with a pathological fracture of the femur due to a diffuse haemangioma of the lower limb. Open reduction and internal fixation with a dynamic compression plate were carried out after heparinisation and transfusion with washed blood cells, fresh-frozen plasma and cryoprecipitate. The blood loss was $5000 \mathrm{ml}$, requiring a massive blood transfusion after operation. We believe that open reduction is viable when the general condition of the patient is satisfactory and massive transfusion is possible.

Yamamoto et $\mathrm{al}^{2}$ treated a 23-year-old man with a fracture of the tibial shaft and a diffuse haemangioma of the lower limb complicated by disseminated intravascular coagulation, by immobilisation in a cast and transfusion. Surgical intervention was not attempted in order to avoid massive blood loss. The fracture united after two years.

Menendez and Thommen ${ }^{1}$ described a 43-year-old woman who presented with Kasabach-Merritt syndrome complicated by fracture of the femoral shaft. KasabachMerritt syndrome is a consumptive coagulopathy associated with a massive haemagioma. Platelet consumption is caused by their sequestration into sinusoids and epitheloid chambers of large haemangiomas. Secondary fibrinolysis occurs with thrombocytopenia, hypofibrinogenaemia and increased fibrin degradation products which cause disseminated intra-vascular coagulation. Closed reduction was followed by immobilisation in a hip spica. However, she was unable to tolerate the hip spica. The fracture remained ununited. She refused amputation.

In one of our patients (case 1) the fracture failed to unite after external fixation for six months and autologous bone grafting and hydroxyapatite were used.

In the other patient (case 2), percutaneous injections of bone marrow were administered because of both massive blood loss during previous surgery and the age of the patient. A number of experimental and retrospective clinical studies have shown the benefits of this form of treatment in both acute fractures and nonunions. ${ }^{5-7}$ The method is safe, simple and requires less time than autologous bone grafting. A recent prospective, randomised investigation of closed fractures of the tibial shaft has shown the benefits of percutaneous injection of marrow. ${ }^{8}$ We used a $21 \mathrm{G}$ needle under local anaesthesia. There was little bleeding.

The options for treatment for fractures due to a haemangioma are complex. Internal fixation is possible when bleeding can be controlled. When internal fixation is hindered by large or diffuse haemangiomas, or when the general condition of the patient is poor, a cast or external fixation offers an alternative. However, bony union is slow but may be accelerated by the injection of bone marrow.

No benefits in any form have been received or will be received from a commercial party related directly or indirectly to the subject of this article.

\section{References}

1. Menendez LR, Thommen VD. Kasabach-Merritt syndrome complicating treatment of a closed femoral fracture. Clin Orthop 1995;316:185-8.

2. Yamamoto H, Muneta T, Asahina S, Furuya K, Suzuki K. Lower leg fracture with Parkes-Weber syndrome complicated by disseminated intravascular coagulation. J Orthop Trauma 1995;9:449-52.

3. Ezawa M, Abe T, Ogawa K, et al. Hemangiomatosis of bone with repeated pathological fractures: a case report. Orthopedic Surgery (Seikei Geka, in Japanese) 1999;50:1107-9.

4. Chen CE, Ko JY, Wang CJ. Kasabach-Merritt syndrome complicating the treatment of a femoral shaft in adult. J Trauma 2002;52:789-92.

5. Garg NK, Gaur S, Shama S. Percutaneous autogenous bone marrow grafting in 20 cases of ununited fracture. Acta Orthop Scand 1993;64:671-2.

6. Connolly JF. Injectable bone marrow preparation to stimulate osteogenic repair. Clin Orthop 1995;313:8-18.

7. Nade $\mathbf{S}$. Osteogenesis after bone and bone marrow transplantation. II: the initial cellular events following transplantation of decalcified allografts of cancellous bone. Acta Orthop Scand 1977;48:572-6.

8. Khanal GP, Garg M, Singh GK. A prospective randomized trial of percutaneous marrow injection in series of closed fresh tibial fractures. Int Orthop 2004;28:167-70, 